\title{
Time-Domain Equivalent Edge Currents for Transient Scattering
}

\author{
Ayhan Altıntaş, Senior Member, IEEE, and Peter Russer, Fellow, IEEE
}

\begin{abstract}
Time-domain equivalent edge currents (TD-EEC) are developed for the transient scattering analysis. The development is based on the Fourier inversion of frequency domain equivalent edge current expressions. The time-domain diffracted fields are expressed in terms of a contour integral along the diffracting edges for any arbitrary input pulse shape, thereby yielding finite results at the caustics of diffracted rays. The approach also eliminates the need for the evaluation of a convolution integral in the time domain geometrical theory of diffraction (GTD) analysis. The results are compared with the first order GTD results for the transient scattering analysis for a circular disk.
\end{abstract}

Index Terms-Electromagnetic transient scattering, equivalent edge currents, high-frequency techniques in electromagnetics, time-domain methods.

\section{INTRODUCTION}

$\mathbf{F}$ OURIER inversion of high-frequency fields gives a clear picture of the scattering mechanism when the object size is large compared to the wavelength at the lowest frequency considered. Ray optical techniques have been commonly applied for the determination of scattered fields at high frequencies [1]-[3]. Equivalent edge currents are employed when the ray optics fail due to the observation point being at or around the caustics of the rays, or even when there is no ray reaching the observation. In addition, they are useful when the input field has spatial variations along the edge [4].

Recently, direct use of physical optics (PO), geometric theory of diffraction (GTD) and uniform theory of diffraction (UTD) in the time domain has been of interest [5]-[7]. The advantages are several. Efficient and faster computation, more suitable solutions when the pulse width is narrow compared with the geometrical dimensions of the scattering object, feasibility to implement a hybrid solution by combination with various numerical time domain methods such as finite difference time domain (FDTD) and TLM. As in the frequency domain, the problem of PO is the limited accuracy yet the simplicity makes it still a desirable approach in certain applications. The time-domain GTD and its extensions have better accuracy and are suitable for multiple diffraction analysis, however, in addition to becoming invalid at caustics of diffracted rays, they require a convolution integral of the input pulse with the so-called "time-domain diffraction coefficients." In the proposed time-domain equivalent current approach, diffracted fields are obtained by an integration of

Manuscript received February 11, 1999; revised June 20, 2000.

A. Altıntaş is with the Department of Electrical and Electronics Engineering, Bilkent University, 06533 Ankara, Turkey.

P. Russer is with the Institut für Hochfrequenztechnik, Technische Universität München, D-80333 Munich, Germany.

Publisher Item Identifier S 0018-926X(01)03183-0. the input pulse over the edge contour and thereby remain finite at caustic regions. In addition, arbitrary pulse inputs can be applied without further processing. When the optical distance is stationary at discrete points over the edge contour satisfying generalized Fermat's principle (the observation point being on the Keller cone), the time-domain equivalent edge currents (TD-EEC) integration recovers the GTD ray optical solution. The approach will, therefore, prove to be useful especially for the transient scattering analysis from plate structures. It is noted that the accuracy of high-frequency based solutions is increased if the low-frequency content of the input pulse is weak [2].

This paper deals with the development of the TD-EEC for transient scattering applications. First, a brief review of the timedomain GTD is given. Followed by a description of the TD-EEC approach. Finally, transient scattering from a circular disk is analyzed using the TD-EEC and the results are compared with the Fourier inversion of the GTD analysis. It is noted that a time-domain version of the physical theory of diffraction is reported very recently [8], however, the numerical results are presented only for two-dimensional (2-D) structures.

\section{BACKGROUND}

For scatterers with edges, the geometrical optical incident and reflected rays are complemented with the edge-diffracted fields of the GTD or the UTD. Analytical time-domain solutions based on the GTD and the UTD are obtained by the Fourier inversion of the corresponding GTD and UTD diffraction coefficients. These are called the "time-domain diffraction coefficients." For an impulse excitation, the scattered fields are proportional to the time-domain diffraction coefficients. However, for a general pulse excitation, a convolution integral of the excitation with the time-domain diffraction coefficients is required to get the scattered field as follows:

$$
\begin{aligned}
& \mathbf{e}_{d}^{G T D}(s, t)=A(s) \int_{t_{0}}^{t-(s / c)} \mathbf{e}^{i n c}\left(Q, t^{\prime}\right) \\
& \cdot \overline{\bar{D}}\left(t-\frac{s}{c}-t^{\prime}\right) d t^{\prime}
\end{aligned}
$$

where

$s \quad$ distance from the diffraction point $Q$ to the observation;

c speed of light;

$t_{0} \quad$ time when the excitation $\mathbf{e}^{i n c}$ reaches $Q$;

$A(s)$ spreading factor for the diffracted rays;

$\overline{\bar{D}}(t)$ time-domain dyadic diffraction coefficient obtained by the inverse transform of frequency domain dyadic diffraction coefficient. 


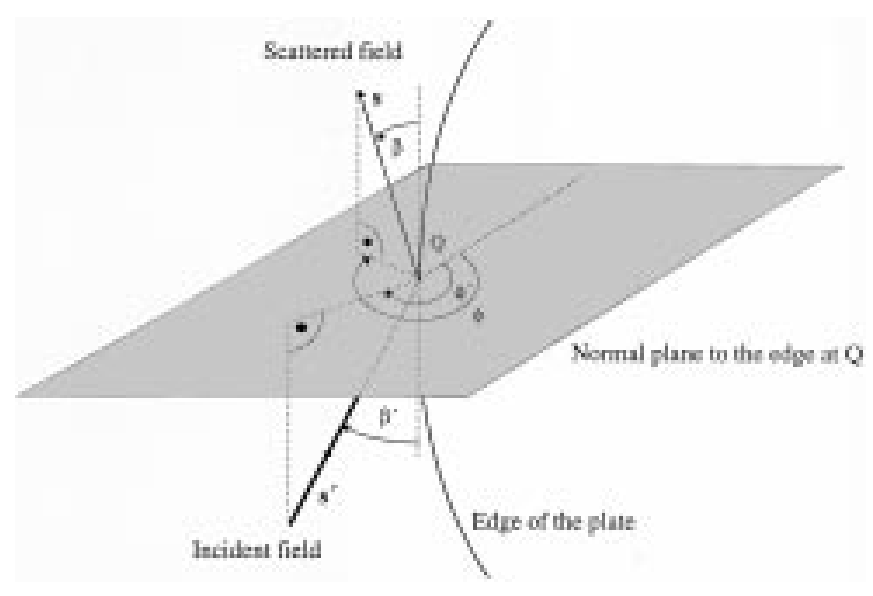

Fig. 1. Definition of the diffraction angles for the edge of a plate.

The expressions for $\overline{\bar{D}}(t)$ are given as

$$
\overline{\bar{D}}(t)=-\hat{\beta}^{\prime} \hat{\beta} D_{s}(t)-\hat{\phi}^{\prime} \hat{\phi} D_{h}(t)
$$

where $D_{s, h}$ are the soft and hard diffraction coefficients and the unit vectors $\hat{\beta}^{\prime}, \hat{\beta}, \hat{\phi}^{\prime}, \hat{\phi}$ are defined in the increasing angle directions of the corresponding diffraction angles as shown in Fig. 1. The angles $\beta^{\prime}$ and $\beta$ are measured from the edge tangent to the incident and diffracted ray directions, respectively. The angles $\phi^{\prime}$ and $\phi$ are measured on the normal plane to the edge by projecting the incident and diffracted ray directions, respectively. The time-domain diffraction coefficients of the GTD for a plate structure is given as

$$
\begin{aligned}
D_{s, h}(t)=\sqrt{\frac{c}{2 t}} & \frac{-1}{2 \pi \sin \beta} \\
& \cdot\left[\frac{1}{\cos \left(\frac{\phi-\phi^{\prime}}{2}\right)} \mp \frac{1}{\cos \left(\frac{\phi-\phi^{\prime}}{2}\right)}\right] .
\end{aligned}
$$

For the UTD, the four term diffraction coefficient is considered. Each term contains a transition function with a frequency dependent length parameter. Fourier inversion of each term can be taken analytically to get the time-domain UTD diffraction coefficient [6], [7]. The time-domain diffraction coefficients are applied at each of the diffraction points satisfying the generalized Fermat's principle. For this reason, time-domain ray optical solutions are not free from their caustic-related difficulties in the frequency domain.

\section{Time-Domain EQuivalent Edge CuRRents}

Equivalent currents have been developed in the frequency domain in order to overcome some of the difficulties of the ray optical solutions. The purpose is to replace the sharp edges with equivalent electric and magnetic line currents so that their radiation will give the far zone high frequency scattered field from the edges. Consider the time-harmonic scattering configuration

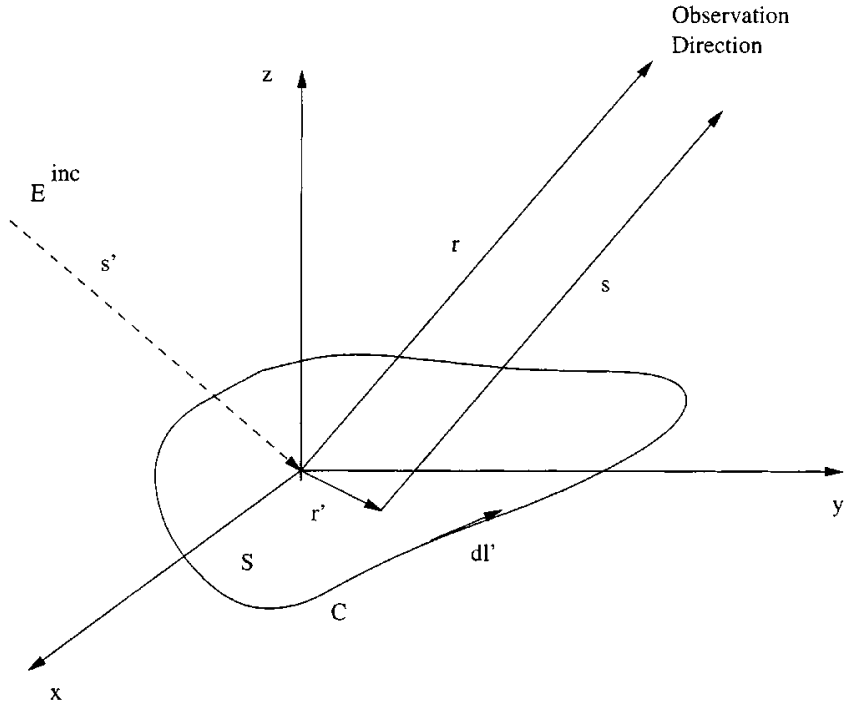

Fig. 2. Scattering geometry for a plate on the $x-y$ plane.

for a flat plate in Fig. 2. The scattered far field is written as the radiation integral

$$
\mathbf{E}^{s}(r, \omega)=j k Z_{o} \iint_{S} \hat{\mathbf{s}} \times \hat{\mathbf{s}} \times \mathbf{J} \frac{e^{-j k s}}{4 \pi s} d S^{\prime}
$$

where

J induced surface current on the plate;

$k$ free-space wavenumber;

$s \quad$ distance from the source to the observation;

$\hat{\mathbf{s}} \quad$ unit vector along $s$.

Following [9], one can obtain an expression for the scattered field in terms of equivalent electric and magnetic currents flowing along the edge as follows:

$$
\begin{aligned}
\mathbf{E}^{s}(r, \omega) & =\frac{j k Z_{o}}{4 \pi} \frac{e^{-j k r}}{r} \\
& \oint_{C} \cdot\left[\hat{\mathbf{s}} \times \hat{\mathbf{s}} \times \hat{l} I_{\omega}+Z_{o}^{-1} \hat{\mathbf{s}} \times \hat{l} M_{\omega}\right] e^{j \mathbf{k} \cdot \mathbf{r}^{\prime}} d l^{\prime}
\end{aligned}
$$

where $r$ is the far-zone distance, $\mathrm{r}^{\prime}$ is the position vector along the edge. The length element along the edge is given by $\overline{d l}^{\prime}=$ $\hat{l} d l^{\prime}$. The expressions for the equivalent curents $I_{\omega}$ and $M_{\omega}$ are determined by applying asymptotic approaches. The final result can be expressed as

$$
I_{\omega}\left(\mathbf{r}^{\prime}, \omega\right)=\frac{1}{j k}\left[Z_{o}^{-1} D_{e}^{I} \mathbf{E}^{i n c}\left(\mathbf{r}^{\prime}, \omega\right) \cdot \hat{l}+D_{h}^{I} \mathbf{H}^{i n c}\left(\mathbf{r}^{\prime}, \omega\right) \cdot \hat{l}\right]
$$

and

$$
M_{\omega}\left(\mathbf{r}^{\prime}, \omega\right)=\frac{Z_{o}}{j k} D_{h}^{M} \mathbf{H}^{i n c}\left(\mathbf{r}^{\prime}, \omega\right) \cdot \hat{l}
$$

where $\mathbf{E}^{i n c}, \mathbf{H}^{i n c}$ are the incident fields and $D_{e}^{I}, D_{h}^{I}, D_{h}^{M}$ are angular coefficients depending solely on the four angles $\left(\beta^{\prime}, \phi^{\prime}, \beta\right.$, and $\left.\phi\right)$ on each point of the edge. Over the years, various expressions for the angular coefficients appearing in (7) based on different assumptions and approaches are proposed. The earlier approaches were based on ray-optical 
fields and heuristic extensions. Later, derivations are based on the asymptotic integration of exact currents on the half-plane problem. A better understanding has been achieved by treating PO and nonuniform (fringe) parts of these currents separately yielding [10]-[12]

$$
\begin{aligned}
D_{e}^{I} & =D_{e}^{I, P O}+D_{e}^{I, f} \\
D_{h}^{I} & =D_{h}^{I, P O}+D_{h}^{I, f} \\
D_{h}^{M} & =D_{h}^{M, P O}+D_{h}^{M, f} .
\end{aligned}
$$

Asymptotic integration is then done in the most suitable way for each of the PO and fringe contributions. The angular coefficients for the PO contribution obtained through this approach is given in (11)-(13) at the bottom of the page [13], [14].

The coefficients for the nonuniform or fringe current contribution are given in (14)-(16) at the bottom of the page [4] where

$$
\begin{aligned}
\gamma= & {\left[\left(\sin \beta^{\prime}\left(\sin \beta^{\prime}-\sin \beta \cos \phi\right)\right.\right.} \\
& \left.\left.-\cos \beta^{\prime}\left(\cos \beta-\cos \beta^{\prime}\right)\right) / 2\right]^{1 / 2}
\end{aligned}
$$

and

$$
\begin{aligned}
\zeta= & \cot \beta^{\prime}\left[\sin \beta \cos \phi+\cot \beta^{\prime}\left(\cos \beta-\cos \beta^{\prime}\right)\right] \\
& -\sin \beta^{\prime} \cot \beta \cos \phi .
\end{aligned}
$$

The same idea can be applied in the time domain by taking the inverse Fourier transform of (5) as follows:

$$
\begin{aligned}
\mathbf{e}_{d}^{E E C}(r, t)= & \frac{Z_{o}}{4 \pi r c} \hat{\mathbf{s}} \times \hat{\mathbf{s}} \times \frac{\partial}{\partial t} \oint_{C} I\left(\mathrm{r}^{\prime}, \tau\right) \hat{l} d l^{\prime} \\
& +\frac{1}{4 \pi r c} \hat{\mathbf{s}} \times \frac{\partial}{\partial t} \oint_{C} M\left(\mathrm{r}^{\prime}, \tau\right) \hat{l} d l^{\prime}
\end{aligned}
$$

where $\tau=t-(r / c)+\left(\mathbf{r}^{\prime} \cdot \hat{s} / c\right), I$ and $M$ are the time-domain equivalent electric and magnetic line currents, respectively. By examining the expressions in (6) and (7), one obtains

$$
\begin{aligned}
I\left(\mathbf{r}^{\prime}, \tau\right)= & \int_{-\infty}^{\tau} c\left[Z_{o}^{-1} D_{e}^{I} \mathbf{e}^{i n c}\left(\mathbf{r}^{\prime}, t^{\prime}\right)\right. \\
& \left.\cdot \hat{l}+D_{h}^{I} \mathbf{h}^{i n c}\left(\mathbf{r}^{\prime}, t^{\prime}\right) \cdot \hat{l}\right] d t^{\prime}
\end{aligned}
$$

and

$$
M\left(\mathbf{r}^{\prime}, \tau\right)=\int_{-\infty}^{\tau} c\left[Z_{o} D_{h}^{M} \mathbf{h}^{i n c}\left(\mathbf{r}^{\prime}, t^{\prime}\right) \cdot \hat{l}\right] d t^{\prime}
$$

where $\mathbf{e}^{i n c}$ and $\mathbf{h}^{i n c}$ are the time-domain incident fields. According to (20) and (21) the determination of equivalent edge currents in the time-domain require an integration over time. For general input waveforms the integrals should be taken numerically; however, in the case of computation of the far-zone scattered fields this integration is not needed since the substitution of (20) and (21) into the expression (19) gives

$$
\begin{aligned}
\mathbf{e}_{d}^{E E C}(r, t)= & \frac{1}{4 \pi r} \hat{\mathbf{s}} \times\left[\hat{\mathbf{s}} \times \oint_{C}\left[D_{e}^{I} \mathbf{e}^{i n c}\left(\mathbf{r}^{\prime}, \tau\right)\right.\right. \\
& \left.\cdot \hat{l}+Z_{o} D_{h}^{I} \mathbf{h}^{i n c}\left(\mathbf{r}^{\prime}, \tau\right) \cdot \hat{l}\right] \hat{l} d l^{\prime} \\
& \left.+\oint_{C} Z_{o} D_{h}^{M}\left(\mathbf{h}^{i n c}\left(\mathbf{r}^{\prime}, \tau\right) \cdot \hat{l}\right) \hat{l} d l^{\prime}\right]
\end{aligned}
$$

in which the time retardation is apparent in the definition of $\tau$. The simplicity of this expression is quite appealing. It is a contour integral of the incident field with proper delays and angular coefficients similar to the expression in the frequency domain. It is also noted that the optical distance between the source and observation varies by $\mathbf{r}^{\prime} \cdot\left(\hat{\mathbf{s}}^{\prime}-\hat{\mathbf{s}}\right) / c$ where $\hat{\mathbf{s}}^{\prime}$ is the incoming field direction and it is stationary when $\beta=\beta^{\prime}$. This is the generalized Fermat's principle satisfied by the diffracted rays on the Keller cone where the equivalent edge current integra-

$$
\begin{aligned}
D_{e}^{I, P O} & =-2 \frac{\sin \phi^{\prime}\left(\sin \beta \cos \phi+\sin \beta^{\prime} \cos \phi^{\prime}\right)}{\left[\left(\cos \beta-\cos \beta^{\prime}\right)^{2}+\left(\sin \beta \cos \phi+\sin \beta^{\prime} \cos \phi^{\prime}\right)^{2}\right] \sin \beta^{\prime}} \\
D_{h}^{I, P O} & =2 \frac{\left(\cot \beta \cos \phi+\cot \beta^{\prime} \cos \phi^{\prime}\right)\left(\sin \beta \cos \phi+\sin \beta^{\prime} \cos \phi^{\prime}\right)}{\left[\left(\cos \beta-\cos \beta^{\prime}\right)^{2}+\left(\sin \beta \cos \phi+\sin \beta^{\prime} \cos \phi^{\prime}\right)^{2}\right]} \\
D_{h}^{M, P O} & =2 \frac{\sin \phi\left(\sin \beta \cos \phi+\sin \beta^{\prime} \cos \phi^{\prime}\right)}{\left[\left(\cos \beta-\cos \beta^{\prime}\right)^{2}+\left(\sin \beta \cos \phi+\sin \beta^{\prime} \cos \phi^{\prime}\right)^{2}\right] \sin \beta} .
\end{aligned}
$$

$$
\begin{aligned}
D_{e}^{I, f} & =-4 \frac{\sin \left(\phi^{\prime} / 2\right)\left(\gamma-\sin \beta^{\prime} \cos \left(\phi^{\prime} / 2\right)\right)}{\sin \beta^{\prime}\left[\sin \beta^{\prime}\left(\sin \beta \cos \phi+\sin \beta^{\prime} \cos \phi^{\prime}\right)+\cos \beta^{\prime}\left(\cos \beta-\cos \beta^{\prime}\right)\right]} \\
D_{h}^{I, f} & =-2 \frac{\sin \beta^{\prime}\left[\cot \beta \cos \phi+\cot \beta^{\prime} \cos \phi^{\prime}+\cos \left(\phi^{\prime} / 2\right) \gamma^{-1} \zeta\right]}{\sin \beta^{\prime}\left(\sin \beta \cos \phi+\sin \beta^{\prime} \cos \phi^{\prime}\right)+\cos \beta^{\prime}\left(\cos \beta-\cos \beta^{\prime}\right)} \\
D_{h}^{M, f} & =-2 \frac{\sin \beta^{\prime} \sin \phi\left[1-\sin \beta^{\prime} \cos \left(\phi^{\prime} / 2\right) \gamma^{-1}\right]}{\sin \beta\left[\sin \beta^{\prime}\left(\sin \beta \cos \phi+\sin \beta^{\prime} \cos \phi^{\prime}\right)+\cos \beta^{\prime}\left(\cos \beta-\cos \beta^{\prime}\right)\right]}
\end{aligned}
$$




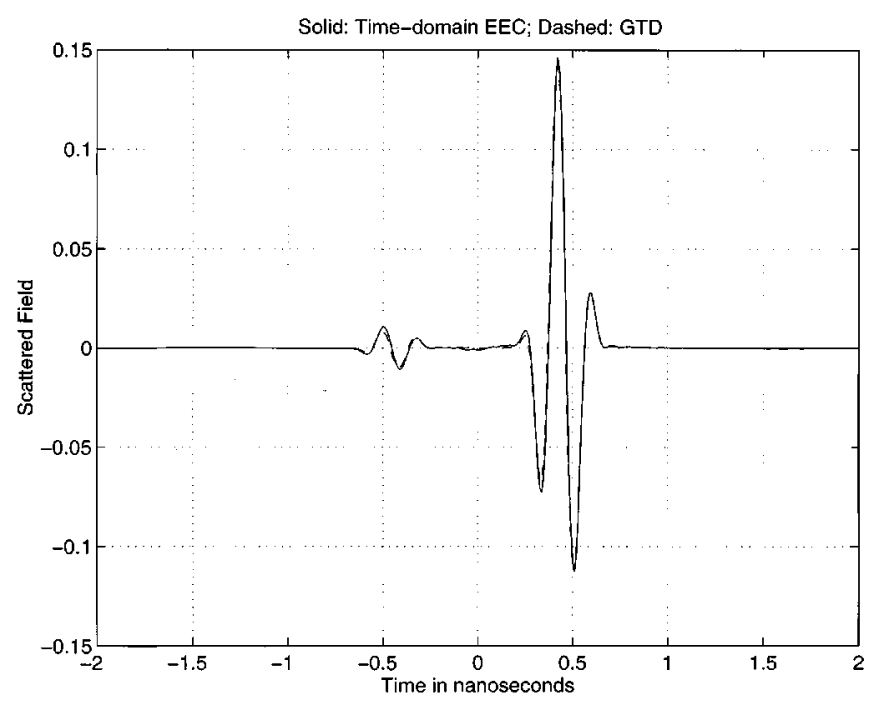

Fig. 3. Comparison of the TD-EEC with the first order GTD for the backscattering from a 3 -in circular disk. $\theta=60^{\circ}, \hat{\theta}$-polarization.

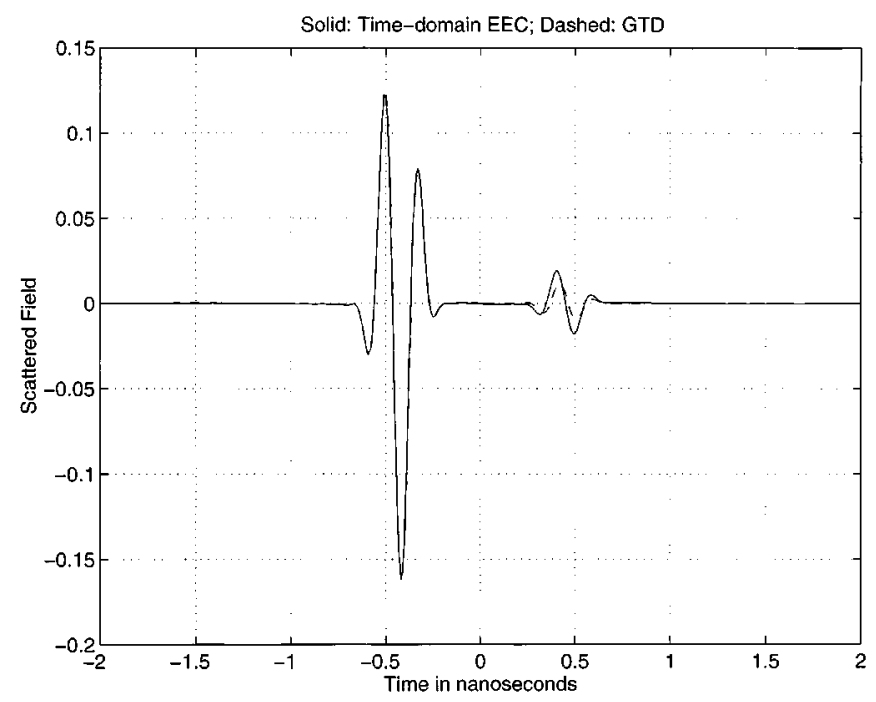

Fig. 4. Comparison of the TD-EEC with the first order GTD for the backscattering from a 3 -in circular disk. $\theta=60^{\circ}, \hat{\Phi}$-polarization.

tion should recover the GTD result. However, the applicability of the TD-EEC is not limited to the existence of the stationary edge points. If the observation point is at or around a ray-optical caustic, or even when stationary points do not exist at all, TD-EEC yield a finite result such as in the case of scattering from polygonal flat plate structures.

\section{NUMERICAL RESULTS}

As a numerical example of the application, transient backscattering from a 3 -in radius circular metallic disk is analyzed using TD-EEC. The results are compared with the Fourier inversion of the first order GTD solution over a frequency range of 1-10 GHz [1]. A Kaiser-Bessel bandpass window (with $\alpha$ parameter being equal to two [15]) has been applied for the elimination of Gibbs phenomenon. For the TD-EEC solution, the input pulse is the Fourier inversion of the windowing function. The disk is assumed to be parallel to the $x y$-plane and the input excitation is in the direction, making an angle $\theta$ from the axis of the disk ( $z$-axis). The unit vectors $\hat{\theta}$ and $\hat{\Phi}$ are in the azimuthal and elevation directions. The comparison of TD-EEC and GTD is shown in Figs. 3 and 4 for $\hat{\theta}$ and $\hat{\Phi}$ polarizations respectively, and almost perfect agreement is worth noting. The GTD solution for this problem is compared with the eigenfunction solution in [1].

\section{CONCLUSION}

Time domain equivalent edge currents for the analysis of transient scattering has been developed. The advantages over the time-domain diffraction coefficient approach have been noted. In addition to applicability at caustic regions, the TD-EEC approach is easily employed for any arbitrary input pulse shape. The approach should prove to be useful when the GTD solution fails due to caustic related problems. The results of TD-EEC are compared with the Fourier inversion of first order GTD results for the transient scattering from a circular disk.

\section{ACKNOWLEDGMENT}

This work was conducted while the first author was on an Alexander von Humboldt Research Fellowship at the Technische Universität München, Munich, Germany. The authors thank Dr. A. K. Dominek for his help and interest in this work.

\section{REFERENCES}

[1] A. K. Dominek, "Transient scattering analysis for a circular disk," IEEE Trans. Antennas Propagat., vol. 39, pp. 815-819, June 1991.

[2] H. Shirai, "Deemphasizing low frequency defects in GTD analysis of pulsed signal scattering by a perfectly conducting flat strip," IEEE Trans. Antennas Propagat., vol. 34, pp. 1261-1266, Oct. 1986.

[3] W. D. Burnside, B. Dewitt, and B. Z. Hollman, "Analysis of TD scattering by a flat plate," IEEE Trans. Antennas Propagat., vol. 33, pp. 917-922, Aug. 1985.

[4] O. Breinbjerg, "EEC analysis of electromagnetic scattering by plane structures," Ph.D. dissertation,, Technical University of Denmark, Dept. Electromagnetic Systems, Mar. 1991.

[5] E. Sun and W. V. T. Rusch, "Time-domain physical-optics," IEEE Trans. Antennas Propagat., vol. 42, pp. 9-15, Jan. 1994.

[6] T. Veruttipong, "Time-domain version of the uniform GTD," IEEE Trans. Antennas Propagat., vol. 38, pp. 1757-1764, Nov. 1990.

[7] P. Rousseau and P. H. Pathak, "Time-domain uniform GTD for a curved wedge," IEEE Trans. Antennas Propagat., vol. 43, pp. 1375-1382, Dec. 1995.

[8] P. M. Johansen, "Time-domain version of the physical theory of diffraction," IEEE Trans. Antennas Propagat., vol. 47, pp. 261-270, Feb. 1999.

[9] A. Michaeli, "Equivalent edge currents for arbitrary aspects of observation," IEEE Trans. Antennas Propagat., vol. 32, pp. 252-258, Mar. 1984.

[10] A. Michaeli, "Elimination of infinities in equivalent edge currents, Part I: Fringe current components," IEEE Trans. Antennas Propagat., vol. 34, pp. 912-918, July 1986.

[11] The Ohio State University, ElectroScience Laboratory, Final Rep. 716 621-1, Apr. 1986.

[12] M. Ando, T. Murasaki, and T. Kinoshita, "Elimination of false singularities in GTD equivalent edge currents," Proc. Inst. Elect. Eng., pt. H, vol. 38, no. 4, Aug. 1991.

[13] T. Oguzer, A. Altintaş, and O. M. Büyükdura, "On the elimination of infinities in the PO component of equivalent edge currents," Wave Motion, vol. 18, no. 1, pp. 1-10, Jan. 1993.

[14] N. Endo, M. Ando, and T. Sekiguchi, "Correction of physical optics by the equivalent edge currents," in 1979 IEEE AP-S Int. Symp., Seattle, USA, pp. 548-551.

[15] F. J. Harris, "On the use of windows for harmonic analysis with the discrete fourier transform," Proc. IEEE, vol. 66, pp. 51-83, Jan. 1978. 


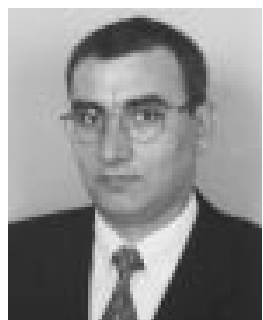

Ayhan Altıntaș (S'82-M'86-SM'93) was born in Turkey on March 29, 1958. He received the B.S. and M.S. degrees from Middle East Technical University, (METU) Ankara, Turkey, and the Ph.D. degree from The Ohio State University, Columbus, in 1979, 1981, and 1986, respectively.

From 1981 to 1987 , he was with the ElectroScience Laboratory, The Ohio State University. From 1995 to 1998, he was an Associate Provost of Bilkent University Ankara, Turkey, where he is currently Professor of Electrical Engineering. He has held various research fellow and guest professor positions at the Australian National University, Canberra, Australia; Tokyo Institute of Technology, Japan; and Technical University of Munich, Germany. His research interests include electromagnetic scattering and propagation, microwaves, fiber and integrated optics.

Dr. Altıntaş is a Fulbright Scholar and an Alexander von Humboldt Fellow. He received the ElectroScience Laboratory Outstanding Dissertation Award 1986 from The Ohio State University, the IEEE 1991 Outstanding Student Branch Counselor Award, the 1991 Research Award of Professor Mustafa N. Parlar Foundation of METU, and Young Scientist Award of Scientific and Technical Research Council of Turkey (Tubitak) in 1996. He is a recipient of the IEEE Third Millennium Medal. He was the Chairman of IEEE Turkey Section for the terms 1991-1993 and 1995-1997. He is a member of Sigma Xi and Phi Kappa Phi.

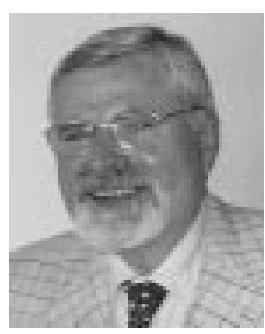

Peter Russer (SM'81-F'94) received the Dipl.-Ing. degree and the Ph.D. degree, both in electrical engineering from the Technische Universität Wien, Austria, in 1967 and 1971, respectively.

From 1968 to 1971, he was with the Technische Universität Wien as an Assistant Professor. In 1971, he joined the Research Institute of AEG-Telefunken, Ulm, Germany, where he worked on fiber optic communication, broadband solid-state electronic circuits, statistical noise analysis of microwave circuits, laser modulation, and fiber-optic gyroscopes. Since 1981, he has been a Professor and Head of the Institute of High Frequency Engineering, Technische Universität München. In 1990, he was a Visiting Professor at the University of Ottawa, Canada, and in 1993, he was a Visiting Professor at the University of Victoria, Canada. From 1992 to March 1995, he was Director of the Ferdinand-Braun-Institut für Höchstfrequenztechnik, Berlin, Germany. His current research interests are electromagnetic fields, integrated microwave and millimeter-wave circuits, statistical noise analysis of microwave circuits, and methods for computer-aided design of microwave circuits. He is author of more than 300 scientific papers in these areas.

Dr. Russer has served on numerous technical program committees and steering committees of various international conferences (IEEE MTT-S, European microwave Conference and as the member of the editorial board of several international journals (Electromagnetics, and International Journal of Numerical Modeling). He is Co-Chairman of U.R.S.I. Commission D. In 1979, he was a co-recipient of the NTG Award for the publication "Electronic Circuits for High Bit Rate Digital Fiber Optic Communication Systems." He is a member of the German Informationstechnische Gesellschaft (ITG) and the German as well as the Austrian Physical Societies. 